\title{
Preliminary Findings from Three Models of Motivational Interviewing Training in Jamaica
}

Henna Budhwani ${ }^{1, *}$ and Sylvie Naar ${ }^{2}$

\begin{abstract}
Introduction: We assessed satisfaction, fidelity, retention, and implementation considerations across three models of motivational interviewing training in Jamaica to identify a promising model for resource-poor settings. Methods: We conducted $t$-tests to assess differences in fidelity and examined qualitative data for barriers and facilitators $(n=52)$.

Results: Only 50-75\% of all models' trainees completed coaching. Model 1 trainees' mean fidelity was 2.83/4.00 compared with Model 3 trainees' at 2.94/4.00 ( $t=-0.710$, confidence interval $=-0.427$ to $0.207, p=0.483$ ). Key barriers to completion and fidelity were lack of funding and time.

Conclusion: We found support for continuing workshop-only trainings; we did not find that higher contact hours produced improved trainee fidelity.
\end{abstract}

Keywords: HIV; Jamaica; Implementation Science; motivational interviewing; youth; capacity building

\section{Introduction}

Motivational interviewing (MI)-when embedded in clinical settings-improves health outcomes across the HIV continuum of care. ${ }^{1-6}$ As such, MI has been included as part of the recommended standard of care for youth living with HIV (YLWH) in the United States. ${ }^{2-6}$ Training providers in MI and supporting them to attain fidelity can be time intensive and cost$1 y,{ }^{7-10}$ and characteristics of organizational settings can act as barriers to implementing MI with fidelity. ${ }^{11}$ Barriers may be exacerbated in resource-constrained settings, but we know that when interventionists are able to culturally tailor models for local contexts, then levels of training satisfaction are higher, and trainees are likely to be more adherent to protocols, which then may lead to higher fidelity outcomes, ultimately leading to improved health equity. ${ }^{12}$

Jamaica is a resource-constrained nation struggling with a concentrated HIV epidemic among youth.
Jamaica has the second highest HIV prevalence in the Caribbean with a staggering $32 \%$ in young men who have sex with men. ${ }^{13-16}$ AIDS is a leading cause of death in Jamaican youth; only $21 \%$ of Jamaican YLWH are on antiretroviral therapy, and a mere 5\% achieve viral suppression. ${ }^{17-20}$ Experts partially attribute these failures to stigmatizing attitudes in health care settings toward YLWH. ${ }^{21-24}$ Past interventions have failed in changing behaviors, in part, because youth are unwilling to disclose their risk behaviors due to stigma emerging in patient-provider communications. ${ }^{11}$ If a model of MI delivery can be refined to promote fidelity, such a model has the potential to improve the in-clinic experiences of Jamaican YLWH.

Considering structural barriers alongside the potential positive impact of MI on Jamaica's HIV continuum of care, our team conducted a pilot study with the objectives of (1) reporting on trainee fidelity and retention, (2) assessing for a significant difference in fidelity

\footnotetext{
${ }^{1}$ University of Alabama at Birmingham (UAB), School of Public Health, Department of Health Care Organization and Policy, Birmingham, Alabama, USA.

${ }^{2}$ Florida State University (FSU), College of Medicine, Center for Translational Behavioral Science, Tallahassee, Florida, USA.

*Address correspondence to: Henna Budhwani, PhD, MPH, Health Care Organization and Policy, University of Alabama at Birmingham, $330 \mathrm{C}$ Ryals Public Health Building, 1665 University Boulevard, Birmingham, AL 35294, USA, E-mail: budhwani@uab.edu
} 
attained by trainees who participated in the lowest contact hour model compared with the highest contact hour model, and (3) characterize barriers and facilitators to MI implementation in Jamaica. ${ }^{13-16}$

\section{Methods}

\section{Data and sample}

Participants $(n=52)$ were HIV providers and prevention and outreach workers in Jamaica who had at least $4 \mathrm{~h}$ of HIV-related client contact per week (2015-2017). Most providers had some high school level education, and a smaller proportion had collegelevel educational attainment. All participants were adults (aged $>18$ years). Participants were identified through consultation with the Jamaica Ministry of Health and the Caribbean Vulnerable Communities Coalition. Randomization was not employed. Trainees only participated in one model. Study participants were not provided any financial incentive.

\section{Ethics}

Approval was provided by the Florida State University Institutional Review Board (\#00000287).

\section{Framework}

We applied the Exploration Preparation Implementation Sustainment (EPIS) framework ${ }^{25}$ to inform the design of this study and used the framework to classify the inner and outer contexts affecting implementation.

\section{Models}

Model 1 included a 2-day face-to-face workshop $(12 \mathrm{~h})$ conducted by American, Motivational Interviewing Network of Trainers (MINTs) followed by six individual MI coaching sessions ( $1 \mathrm{~h}$ each) conducted through Skype or phone call. Model $1(n=20)$ included a maximum of 18 hours of contact. Model 2 reduced the number of coaching sessions from six to four and added two face-to-face booster workshops (6 hours of contact per booster). Model $2(n=20)$ included 28 hours of contact. Model 3 included a 3-day workshop (18 h), two face-to-face booster, two oneon-one coaching sessions, and was conducted by Jamaican facilitators. Model $3(n=12)$ included 32 hours of contact. See Table 1 for features of each model $(n=52)$.

\section{Measures: Workshop satisfaction}

Written pre- and postworkshop satisfaction assessments (quantitative and qualitative) were conducted
Table 1. Features of Three Models of Motivational Interviewing Training in Jamaica

\begin{tabular}{lccc}
\hline & Model 1 & Model 2 & Model 3 \\
\hline No. of workshop attendees & 20 & 20 & 12 \\
American MINTs & Yes & Yes & No \\
Jamaican MINT & No & No & Yes \\
Jamaica facilitators & No & Yes & Yes \\
Nonprovider stakeholders & Yes & No & No \\
Providers & Yes & Yes & Yes \\
Workshop length & $12 \mathrm{~h}$ & $12 \mathrm{~h}$ & $18 \mathrm{~h}$ \\
1-on-1 coaching & Yes & Yes & Yes \\
No. of coaching sessions & 6 & 4 & 2 \\
Boosters workshops & No & Yes & Yes \\
\hline
\end{tabular}

MINT, Motivational Interviewing Network of Trainer.

on paper. Satisfaction was assessed by questions relating to whether topics were relevant, training was useful, trainers were knowledgeable, and whether trainees felt more confident in the delivery MI. Participants were also asked to provide written feedback postworkshop and postbooster (when applicable).

\section{Measures: Fidelity}

MI fidelity was measured through the conduct and coding of standard patient roleplays using the MI coach rating scale (MI-CRS). ${ }^{12}$ Trainees participated in audiorecorded standard patient roleplays, which were scored after the session. The MI-CRS was developed using item response theory and includes 12 items assessing competence on a 4-point Likert scale ranging from poor to excellent. ${ }^{12}$ Competency thresholds are beginner $(<2.0)$, novice $(2.0-2.6)$, intermediate $(2.7-3.3)$, and advanced $(>3.3){ }^{15}$

\section{Analyses}

We conducted an independent samples $t$-test using SPSS 25 to assess whether there were statistical differences in mean fidelity between the highest and lowest contact hour models. To classify implementation barriers and facilitators, we leveraged elements of the thematic analysis approach. Owing to the limited nature of our data, we only identified themes and then assigned those themes as being either a barrier or facilitator, thereafter as being embedded within the inner or outer context, as defined by the EPIS framework. ${ }^{25}$

\section{Results}

Satisfaction

Overall workshop satisfaction was high across all models. Satisfaction ranged across measures and models from $75 \%$ to $100 \%$ were satisfied. 
Table 2. Blinded Motivational Interviewing Fidelity Results from Model $1(n=20)$

\begin{tabular}{lcccc}
\hline Trainee & $\begin{array}{c}\text { No. of coaching } \\
\text { sessions }\end{array}$ & $\begin{array}{c}\text { Most recent } \\
\text { fidelity score }\end{array}$ & $\begin{array}{c}\text { Highest } \\
\text { fidelity score }\end{array}$ & $\begin{array}{c}\text { Average } \\
\text { fidelity score }\end{array}$ \\
\hline A & 0 & 0.00 & 0.00 & 0.00 \\
B & 0 & 0.00 & 0.00 & 0.00 \\
C & 0 & 0.00 & 0.00 & 0.00 \\
D & 1 & 3.33 & 3.33 & 3.33 \\
E & 1 & 2.92 & 2.92 & 2.92 \\
F & 1 & 2.67 & 2.67 & 2.67 \\
G & 1 & 2.25 & 2.25 & 2.25 \\
H & 1 & 3.08 & 3.08 & 3.08 \\
I & 1 & 3.08 & 3.08 & 3.08 \\
J & 1 & 2.67 & 2.67 & 2.67 \\
K & 3 & 3.00 & 3.08 & 2.94 \\
L & 3 & 2.50 & 2.83 & 2.58 \\
M & 4 & 3.08 & 3.08 & 2.23 \\
N & 4 & 2.67 & 2.67 & 2.50 \\
O & 4 & 2.75 & 2.75 & 2.56 \\
P & 5 & 2.50 & 2.83 & 2.68 \\
Q & 5 & 3.58 & 3.58 & 3.22 \\
R & 6 & 3.92 & 3.92 & 3.38 \\
S & 6 & 3.50 & 3.50 & 2.97 \\
T & 6 & 3.67 & 3.67 & 2.99 \\
\hline
\end{tabular}

\section{Model 1: Workshop + coaching + American}

The following statement aptly summarized participant sentiments after the initial workshops:

"Initially, I thought it was a joke to attend the workshop. But coming and reflecting on the skillset, it is needed in my job and will enhance my skill and transform my approach in doing things... I appreciate the opportunity. It was not boring and was creative."

Although satisfaction was high, coaching completion was low. Sixty percent of Model 1's participants entered coaching; $50 \%$ completed three or more coaching sessions $(n=10)$. The mean number of coaching sessions was 3.18 (standard deviation $[\mathrm{SD}]=2.04$ ) sessions. The mean participant fidelity score was 2.83 ( $\mathrm{SD}=0.34$, intermediate competency). See Table 2 for results from Model 1.

Model 2:

Workshop + coaching + boosters + American

Barriers were uncovered and addressed during the adaptation of Model 1 to Model 2, including the in- ability to record live interactions with patients (shifted to using standard patient roleplays), difficultly using the scheduling software (used e-mail to schedule outside of the system), and unstable Internet (provided phone cards for international calling). Considering cultural contexts, we modified the postworkshop process to reduce the coaching sessions from six to four while adding two face-to-face boosters. Feedback solicited during the booster sessions was positive:

"[MI] allows me confidence and control in unknown client situations; provide clarity in connecting client care, client needs and goal aimed at HIV treatment."

Even with this positive feedback, in Model 2 $(n=20)$ coaching attrition stayed the same with $\sim 50 \%$ of trainees completing at least three sessions. Six workshop attendees (30\%) did not complete any coaching. Fourteen trainees $(70 \%)$ scheduled a coaching session but then did not show up. The mean number of coaching sessions of those who completed at least one coaching session was $3.29(\mathrm{SD}=1.10)$ sessions. Attendance in the face-to-face booster sessions was strong with about two-thirds of trainees attending these sessions.

\section{Model 3:}

\section{Workshop+ coaching + boosters + Jamaican}

Model 3 was conducted by Jamaican trainers $(n=12)$; the workshop was extended from 2 to 3 days. Two face-to-face boosters were retained from Model 2, plus two one-on-one coaching sessions. The mean of each participant's average fidelity score was $2.94(\mathrm{SD}=0.54)$, indicating intermediate competency. A trainee comment from Model 3 was that:

\footnotetext{
"Everyone appreciated the presentation style as it allowed for participation; it was experiential. I had no need to worry. Appreciated being stretched out of my comfort zone... I believe it will be useful. I spoke yesterday with my husband about MI and gave the example of when the client says, 'I don't know.' It was an epiphany for me..."
}

Table 3. Inner and Outer Contexts Potentially Influencing Intervention Fidelity

\begin{tabular}{|c|c|c|}
\hline & Barriers & Facilitators \\
\hline Inner contexts & $\begin{array}{l}\text { Funding to support trainee time } \\
\text { Insufficient broadband access in some agencies } \\
\text { Inability to access phones or computers for ongoing coaching }\end{array}$ & $\begin{array}{l}\text { Organization leadership support } \\
\text { Culture of quality improvement } \\
\text { Sufficient staffing to delivery of HIV care to youth }\end{array}$ \\
\hline Outer contexts & $\begin{array}{l}\text { Sociopolitical environment that stigmatizes YLWH, so that trainees } \\
\text { may not be invested in the intervention } \\
\text { Shifting funding priorities } \\
\text { Incentive expectations set by other American and European } \\
\text { capacity building groups }\end{array}$ & $\begin{array}{l}\text { Ongoing coaching } \\
\text { MI fidelity monitoring } \\
\text { Use to expert MI trainers to deliver content } \\
\text { and coaching. }\end{array}$ \\
\hline
\end{tabular}

MI, motivational interviewing; YLWH, youth living with HIV. 


\section{Comparing Model 1 with Model 3 outcomes}

Model 1 included the fewest contact hours and Model 3 included the most contact hours. About 50\% of Model 1's trainees completed their coaching; comparatively, about $75 \%$ of Model 3's trainees completed their coaching. Trainees from both attained, on average, intermediate level of competency. The average fidelity score for Model 1 trainees was 2.83 compared with 2.94 for Model 3 trainees $(t=-0.710$, confidence interval $=-0.427$ to $0.207, p=0.483)$, indicating no statistically significant difference.

\section{Inner and outer contexts}

Using the EPIS framework, ${ }^{25}$ we categorized the inner and outer contexts influencing MI delivery in Jamaica for use by public health scholars and practitioners who conduct intervention research or seek to build capacity by delivering MI Table 3 .

\section{Discussion}

We found some evidence to support the value of continuing to deliver workshop-only trainings (current standard), but we did not find evidence to support generally held belief that higher face-to-face contact models produce higher fidelity.

\section{Limitations}

Detailed participant demographic data were not collected, so we were unable to examine outcomes by age, gender, etc. Contamination could have led to improved outcomes in Models 2 and 3. Self-selection bias may affect study outcomes; participants who decided to enroll in this study may have held different personal characteristics than those who opted out of participating.

\section{Future research}

Since interventions shown to be effective in the United States may not be translatable without substantial adaptation for local contexts, future research examining the implementation of behavioral interventions in resource-constrained settings continues to be warranted.

\section{Conclusion}

Delivering MI with fidelity in settings affected by concentrated HIV epidemics may be accomplished if structural limitations are considered, but also requires attention to implementation contexts, if health equity is to be attained.

\section{Acknowledgments}

The authors thank our exceptional interventionists, Dr. Salome (Nikki) Cockern (Wayne State University), Ms. Joy Crawford (Eve for Life), and Mr. Maurice Bulls (Behavior Change Consulting), as well as the Caribbean Vulnerable Communities Coalition leadership team, and our participants from Jamaica for their commitment to improving the HIV continuum of care outcomes through the implementation of MI in treatment and prevention settings.

\section{Disclaimer}

The content is solely the responsibility of the authors and does not necessarily represent the official views of the National Institutes of Health or the Sparkman Center.

\section{Author Disclosure Statement}

No competing financial interests exist.

\section{Funding Information}

This study was funded by the University of Alabama at Birmingham (UAB) Sparkman Center for Global Health. Research reported in this publication was supported by the University of Alabama at Birmingham (UAB) Sparkman Center for Global Health and the National Institute of Mental Health (NIMH) of the National Institutes of Health (NIH) under Award Number K01MH116737.

\section{References}

1. Naar-King S, Suarez M. Motivational Interviewing with Adolescents and Young Adults. New York: Guilford Press, 2011.

2. AIDS.Gov—US Department of Health \& Human Services. HIV/AIDS Care Continuum. Available at www.aids.gov/federal-resources/policies/carecontinuum/ Accessed September 29, 2020.

3. Bell K, Cole BA. Improving medical students' success in promoting health behavior change: a curriculum evaluation. J Gen Intern Med. 2008;23: 1503-1506.

4. Lundahl BW, Kunz C, Brownwell C, et al. A meta-analysis of Motivational Interviewing: twenty-five years of empirical studies. Res Soc Work Pract. 2010;20:137-160.

5. Mbuagbaw L, Ye C, Thabane L. Motivational interviewing for improving outcomes in youth living with HIV. Cochrane Database Syst Rev. 2012;9: CD009748.

6. Naar-King S, Outlaw A, Green-Jones $M$, et al. Motivational interviewing by peer outreach workers: a pilot randomized clinical trial to retain adolescents and young adults in HIV care. AIDS Care. 2009;21:868-873.

7. Moyers TB, Martin T, Manuel JK, et al. Assessing competence in the use of motivational interviewing. J Subst Abuse Treat. 2005;28:19-26.

8. Brownson RC, Colditz GA, Proctor EK. Dissemination and Implementation Research in Health: Translating Science to Practice. New York, NY: Oxford University Press, 2012.

9. Scheirer MA, Dearing JW. An agenda for research on the sustainability of public health programs. Am J Public Health. 2011;101:2059-2067.

10. Moyers TB, Manuel JK, Wilson PG, et al. A randomized trial investigating training in Motivational Interviewing for behavioral health providers. Behav Cogn Psychother. 2008;36:149-162. 
11. Amodeo $\mathrm{M}$, Lundgren $\mathrm{L}$, Cohen $\mathrm{A}$, et al. Barriers to implementing evidence-based practices in addiction treatment programs: comparing staff reports on motivational interviewing, adolescent community reinforcement approach, assertive community treatment, and cognitivebehavioral therapy. Eval Prog Plann. 2011;34:382-389.

12. Naar S, MacDonell K, Chapman JE, et al. Testing a Motivational interviewing implementation intervention in adolescent HIV clinics: protocol for a type 3, hybrid Implementation-effectiveness trial. JMIR Res Protoc. 2019;8:e11200.

13. UNAIDS \& Jamaican Ministry of Health. Jamaica Progress Report: Global AIDS Response Progress Report. Available at www.unaids.org/sites/ default/files/country/documents//JAM_narrative_report_2014.pdf. Accessed December 3, 2014.

14. Figueroa JP. The challenge of sexually active school children in the Caribbean in the era of HIV/AIDS. In: Morrissey EM, Bernard M, Bundy D (eds). Challenging HIV \& AIDS: A New Role for Caribbean Education. Kingston, Jamaica: Ian Randle Publishers, UNESCO, 2010, pp.167-173.

15. Sutherland M. The Caribbean's HIV/AIDS epidemic: theory, research and interventions. J HIV/AIDS Infect Dis. 2014;2:1-11.

16. UNAIDS. Report on the Global AIDS Epidemic 2013. 2013. Available at https://www.unaids.org/en/media/unaids/contentassets/documents/ epidemiology/2013/gr2013/UNAIDS_Global_Report_2013_en.pdf Accessed October 2, 2020

17. National HIV/STD Control Program MoH, Kingston, Jamaica. Jamaica HIV/AIDS Epidemic Update Jan-June 2006. Kingston, Jamaica: Jamaica Ministry of Health.

18. UNICEF. Strengthening adolescent component of national HIV programmes through country assessments in Jamaica. 2015. Available at https://www.unicef.org/jamaica/media/401/file/StrengtheningAdolescent-Component-of-National-HIV-Programmes-through-CountryAssessments-in-Jamaica-2015.pdf Accessed January 5, 2019.

19. Harris O. Social and Cultural Determinants of HIV Risk Among Young Jamaican Men Who Have Sex with Men. 2014. [Order No. 3621200]. Available at https://urresearch.rochester.edu/institutionalPublicationPublicView.action?institutionalltemld = 28044 Accessed October 2, 2020.
20. Harvey KM, Carrington D, Duncan J, et al. Evaluation of adherence to highly active antiretroviral therapy in adults in Jamaica. West Indian Med J. 2008;57:293-297.

21. Centers for Disease Control and Prevention. Highlights from the Jamaica Reproductive Health Survey, 2002-03. Available at www.cdc.gov/ reproductivehealth/Surveys/Jamaica.htm. Accessed November 17, 2014.

22. Nyblade L, Stangl A, Weiss E, et al. Combating HIV stigma in health care settings: what works? J Int AIDS Soc. 2009;12:15.

23. Budhwani H, Hearld KR, Barrow G, et al. A comparison of younger and older men who have sex with men using data from Jamaica AIDS Support for Life: characteristics associated with HIV status. Int J STD AIDS. 2016;27: 769-775.

24. Population Reference Bureau. The World's Youth 2006 Data Sheet. Available at www.prb.org/pdf06/WorldsYouth2006Datasheet.pdf Accessed June 1, 2020.

25. Moullin JC, Dickson KS, Stadnick NA, et al. Systematic review of the exploration, preparation, implementation, sustainment (EPIS) framework. Implement Sci. 2019;14:1.

Cite this article as: Budhwani $\mathrm{H}, \mathrm{Naar} \mathrm{S}$ (2020) Preliminary findings from three models of motivational interviewing training in Jamaica, Health Equity 4:1, 438-442, DOI: 10.1089/heq.2020.0034.

$\begin{aligned} & \text { Abbreviations Used } \\ & \mathrm{EPIS}=\text { Exploration Preparation Implementation Sustainment } \\ & \mathrm{MI}=\text { motivational interviewing } \\ & \mathrm{MI}-\mathrm{CRS}=\text { motivational interviewing coach rating scale } \\ & \mathrm{MINT}=\text { Motivational Interviewing Network of Trainer } \\ & \mathrm{SD}=\text { standard deviation } \\ & \mathrm{YLWH}=\text { youth living with HIV }\end{aligned}$

